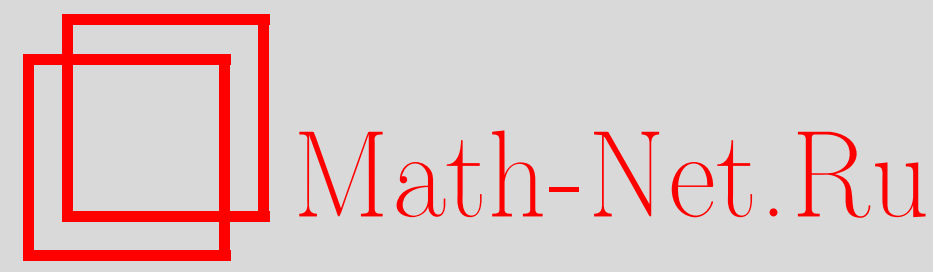

Г. М. Вайникко, Н. В. Лебедева, И. К. Лифанов, Численное решение сингулярного и гиперсингулярного интегральных уравнений на отрезке и дельта-функция, Матем. сб., 2002, том 193, номер 10, 3-16

DOI: https://doi.org/10.4213/sm683

Использование Общероссийского математического портала Math-Net.Ru подразумевает, что вы прочитали и согласны с пользовательским соглашением http://www . mathnet.ru/rus/agreement

Параметры загрузки:

IP: 34.239 .49 .27

26 апреля 2023 г., 15:01:50 


\section{Численное решение сингулярного и гиперсингулярного интегральных уравнений на отрезке и дельта-функция}

\footnotetext{
Предложен вариант метода дискретных вихрей численного решения сингулярного и гиперсингулярного интегральных уравнений первого рода на отрезке в случае, когда в правой части уравнения имеется дельта-функция.

Библиография: 9 названий.
}

В последнее время [1]-[5] в аэродинамике стали активно исследовать механизацию крыльев с использованием эжектирования (отсоса) внешнего потока. В случае плоскопараллельных течений для тонких профилей было показано [2], что задачу с отсосом внешнего потока можно свести к решению сингулярного интегрального уравнения первого рода. Причем из физических соображений следует, что решение полученного интегрального уравнения надо искать в классе функций, имеющих в точке расположения отсоса особенность, интегрируемую в смысле главного значения по Коши. Правая часть этого уравнения является гладкой функцией, определенной всюду на контуре профиля, за исключением точки отсоса (по непрерывности ее можно продолжить на эту точку). При численном решении задачи с эжекцией методом дискретных вихрей расчетные точки выбирают так, чтобы точка отсоса являлась одной из них, и при написании системы линейных алгебраических уравнений то уравнение, что соответствует точке отсоса, пропускается.

Получается система с $n$ неизвестными и $n-1$ уравнениями. Поэтому из тех или иных физических соображений ищется дополнительное уравнение, чтобы получить разрешаемую однозначно систему $n$ уравнений с $n$ неизвестными [3], [4]. Взяв несколько иную физическую трактовку задачи с эжекцией [6], приходим к тому же самому сингулярному интегральному уравнению первого рода, но в правой части этого уравнения появляется дельта-функция с носителем, расположенным в точке отсоса. Оказалось, что наличие дельта-функции в правой части позволяет строить более удобные методы численного решения соответствующих задач аэродинамики.

В настоящей работе предложен вариант метода дискретных вихрей численного решения сингулярного и гиперсингулярного интегральных уравнений первого рода на отрезке в случае, когда в правой части уравнения имеется дельтафункция.

\section{$\S 1$. Характеристическое сингулярное интегральное уравнение на отрезке}

С помощью моделирования вихревым слоем [3] задача обтекания тонкого профиля идеальной несжимаемой жидкостью сводится к решению сингулярного ин-

$$
\text { (C) Г. М. ВАЙНИКко, Н. В. ЛЕБЕДЕвА, И. К. ЛиФАНОВ } 2002
$$


тегрального уравнения на отрезке $[-1,1]$ :

$$
\frac{1}{\pi} \int_{-1}^{1} \frac{\gamma(x) d x}{x_{0}-x}=f\left(x_{0}\right), \quad x_{0} \in(-1,1)
$$

где $f(x)$ является гёльдеровской функцией степени $\alpha$, т.е. $f(x) \in H(\alpha)$ на $[-1,1]$. Если на профиле имеется устройство эжектирования внешнего потока (отсос), которое моделируется стоком в точке $q, q \in(-1,1)$, то при выполнении граничного условия непротекания профиля на его стороне, противоположной размешению отсоса, в правой части уравнения (1.1) появляется [6] дельта-функция с носителем в точке $q$, т.е. функция

$$
f_{\delta, q}\left(x_{0}\right)=Q \cdot \delta\left(x_{0}-q\right), \quad x_{0}, q \in(-1,1),
$$

где $Q$ - некоторое число, а функцию $\delta\left(x_{0}-q\right)$ определим следующим образом:

$$
\begin{aligned}
\delta\left(x_{0}-q\right)= & \begin{cases}0, & x_{0} \neq q, x_{0}, q \in(-1,1), \\
+\infty, & x_{0}=q,\end{cases} \\
& \int_{-1}^{1} \delta\left(x_{0}-q\right) d x_{0}=1 .
\end{aligned}
$$

Функцию $\delta\left(x_{0}-q\right)$ будем представлять как предел:

$$
\begin{aligned}
\delta\left(x_{0}-q\right) & =\lim _{h \rightarrow 0} \delta_{h}\left(x_{0}-q\right), \\
\delta_{h}\left(x_{0}-q\right) & = \begin{cases}\frac{1}{h}, & x_{0} \in\left[q-\frac{h}{2}, q+\frac{h}{2}\right], \\
0, & x_{0} \notin\left[q-\frac{h}{2}, q+\frac{h}{2}\right] .\end{cases}
\end{aligned}
$$

Предположим теперь, что в уравнении (1.1) справа стоит функция $f_{\delta, q}\left(x_{0}\right)$. Используя идеи метода дискретных вихрей [3], [4], [7], построим метод численного решения этого уравнения для различных индексов $\varkappa=1,0,-1$ (см. [8]). Возьмем на $[-1,1]$ два множества точек $E=\left\{x_{k}, k=1, \ldots, n\right\}$ и $E_{0}=\left\{x_{0 j}, j=0,1, \ldots, n\right\}$, удовлетворяющих условиям:

$$
\begin{gathered}
\left|x_{k+1}-x_{k}\right|=h, \quad k=1, \ldots, n-1, \quad h=\frac{2}{n+2}, \\
\left|x_{0 j+1}-x_{0 j}\right|=h, \quad j=0,1, \ldots, n-1, \\
x_{1}-x_{00}=\frac{h}{2}, \quad x_{0 k}=x_{k}+\frac{h}{2}, \quad k=1, \ldots, n, \\
x_{00}+1=h \cdot q_{1, h}, \quad 1-x_{0 n}=h \cdot q_{2, h}, \quad 0<p_{1} \leqslant q_{1, h}, \quad q_{2, h} \leqslant p_{2}<+\infty,
\end{gathered}
$$

где $p_{1}$ и $p_{2}-$ заданные числа и точка $q$ при любом $n$ лежит в множестве $E_{0}, q=x_{0} j_{q}$ (см. [7; $§ 1.3]$, здесь же указано, как эти множества построить).

Вначале рассмотрим решения индекса $\varkappa=1$, т.е. обращающиеся на обоих концах отрезка $[-1,1]$ в бесконечность. Справедлива следующая 
ЛЕмма 1.1. Пусть в правой части уравнения (1.1) стоит функция $f_{\delta, q}\left(x_{0}\right)$, $x_{0}, q \in(-1,1)$. Тогда решение системы линейных алгебрачческих уравнений

$$
\begin{gathered}
\frac{1}{\pi} \sum_{k=1}^{n} \frac{\gamma_{n}\left(x_{k}\right) \cdot h}{x_{0 j}-x_{k}}=Q \cdot \delta_{h}\left(x_{0 j}-q\right), \quad j=1, \ldots, n-1, \quad x_{0 j_{q}}=q, \\
\sum_{k=1}^{n} \gamma_{n}\left(x_{k}\right) \cdot h=0
\end{gathered}
$$

и значение функиии

$$
\gamma(x)=-\frac{1}{\pi} \frac{\sqrt{1-q^{2}}}{1-x^{2}} \cdot \frac{Q}{x-q}
$$

связаны соотношением

$$
\left|\gamma\left(x_{k}\right)-\gamma_{n}\left(x_{k}\right)\right| \leqslant \theta_{n}\left(x_{k}\right), \quad k=1, \ldots, n,
$$

в котором величина $\theta_{n}\left(x_{k}\right)$ удовлетворяет неравенствам:

1) для всех точек $x_{k} \in[-1+\delta, q-\delta] \cup[q+\delta, 1-\delta]$, где $\delta>0$ сколь угодно мало,

$$
\theta_{n}\left(x_{k}\right) \leqslant C_{\delta} \cdot h^{\lambda_{1}}, \quad \lambda_{1}>0,
$$

2) для всех точек $x_{k} \in[-1,1]$

$$
\sum_{k=1}^{n} \theta_{n}\left(x_{k}\right) \cdot h \leqslant C \cdot h^{\lambda_{2}}, \quad \lambda_{2}>0
$$

где $C_{\delta}, C$ - некоторые константьи, не зависящие от $n$.

ДокАЗАТЕЛЬСтво. Применяя результаты [ 7 ; 5.1$]$, получаем, что решение системы (1.8) дается формулой

$$
\begin{gathered}
\gamma_{n}\left(x_{k}\right)=-\frac{1}{h} I_{1, k}^{(n)} \cdot \sum_{j=1}^{n} \frac{1}{h} I_{1,0 j}^{(n)} \cdot \frac{\pi \cdot Q \cdot \delta_{h}\left(x_{0 j}-q\right)}{x_{k}-x_{0 j}} h, \quad k=1, \ldots, n, \\
I_{1, k}^{(n)}=I_{0, k}^{(n)} \cdot \frac{1}{x_{0 n}-x_{k}}, \quad I_{0, k}^{(n)}=\frac{\prod_{m=1}^{n}\left(x_{0 m}-x_{k}\right)}{\prod_{\substack{m=1 \\
m \neq k}}^{n}\left(x_{m}-x_{k}\right)}, \\
I_{1,0 j}^{(n)}=I_{0,0 j}^{(n)} \cdot\left(x_{0 n}-x_{k}\right), \quad I_{0,0 j}^{(n)}=\frac{\prod_{m=1}^{n}\left(x_{0 j}-x_{m}\right)}{\prod_{\substack{m=1 \\
m \neq j}}^{n}\left(x_{0 j}-x_{0 m}\right)} .
\end{gathered}
$$

В силу определения функции $\delta_{h}\left(x_{0}-q\right)$ (см. (1.6)) получаем, что (1.13) примет вид

$$
\gamma_{n}\left(x_{k}\right)=-\frac{1}{h} I_{1, k}^{(n)} \cdot \frac{1}{h} \cdot I_{1,0 j_{q}}^{(n)} \frac{\pi \cdot Q}{x_{k}-x_{0 j_{q}}}, \quad k=1, \ldots, n
$$


Так как справедливы представления $[7 ; \S 5.1]$

$$
\begin{aligned}
& \frac{1}{h} \cdot I_{1, k}^{(n)}=\frac{1}{\pi} \cdot \frac{1}{\sqrt{1-x_{k}^{2}}}+O\left(\frac{h}{\left(1-x_{k}^{2}\right)^{3 / 2}}\right), \quad k=1, \ldots, n, \\
& \frac{1}{h} \cdot I_{1,0 j}^{(n)}=\frac{1}{\pi} \cdot \sqrt{1-x_{0 j}^{2}}+O\left(\frac{h}{\left(1-x_{0 j}^{2}\right)^{1 / 2}}\right), \quad j=1, \ldots, n-1,
\end{aligned}
$$

где $O(\alpha)$ величина порядка $\alpha$, то из формул $(1.14),(1.15)$ получаем (учитьвая, что $\left.x_{0 j_{q}}=q\right)$

$$
\gamma_{n}\left(x_{k}\right)=-\frac{1}{\pi} \frac{\sqrt{1-q^{2}}}{\sqrt{1-x_{k}^{2}}} \cdot \frac{Q}{x_{k}-q}+\theta_{n}^{*}\left(x_{k}\right),
$$

где величина $\left|\theta_{n}^{*}\left(x_{k}\right)\right|=\theta_{n}\left(x_{k}\right)$ удовлетворяет неравенствам (1.11) и (1.12). Лемма 1.1 доказана.

Покажем теперь, что функция $\gamma(x)$ в (1.9) является решением уравнения (1.1), если в правой части стоит функция $f_{\delta, q}\left(x_{0}\right)$, при рассмотрении этого уравнения как оператора в пространствах $H_{\rho}^{\lambda}$ (типа соболевских с весом), построенных в [6; §4].

Напомним вначале понятие пространства $L_{2, \rho}$ действительных функций, квадрат модуля которых интегрируем на $[-1,1]$ с весом $\rho=\rho(x)$, т.е. таких функций $f(x), x \in[-1,1]$, что $\int_{-1}^{1} \rho(x) \cdot|f(x)|^{2} d x<+\infty$, где $\rho(x)>0$ почти всюду на $[-1,1]$. Естественным образом вводится скалярное произведение функций в этом пространстве

$$
(f(x), g(x))=\int_{-1}^{1} \rho(x) f(x) g(x) d x
$$

После этого пространство $L_{2, \rho}$ становится гильбертовьм, и поэтому можно взять полную систему ортогональных функций $\left\{P_{n}(x), n=0,1, \ldots\right\}$, являюшихся базисом в $L_{2, \rho}$, т.е.

$$
\int_{-1}^{1} \rho(x) P_{n}(x) P_{m}(x) d x=\delta_{n}^{m}
$$

где $\delta_{n}^{m}=0, n \neq m$, и $\delta_{n}^{n}=1, m, n=0,1, \ldots,-$ символ Кронекера. Любая функция $f(x) \in L_{2, \rho}$ представляется рядом Фурье по системе этих функций, сходящимся по норме $L_{2, \rho}$ :

$$
\begin{gathered}
f(x)=\sum_{n=0}^{\infty} \widehat{f}(n) P_{n}(x), \quad \widehat{f}(n)=\int_{-1}^{1} \rho(x) f(x) P_{n}(x) d x, \\
\|f\|_{L_{2, \rho}}^{2}=\int_{-1}^{1} \rho(x) f^{2}(x) d x=\sum_{n=0}^{\infty} \widehat{f}^{2}(n)<+\infty .
\end{gathered}
$$

Теперь можно дать следуюшее определение пространства $H_{\rho}^{\lambda}$ (типа соболевского) с весом $\rho$, связанного с пространством $L_{2, \rho}$. 
ОПреДЕЛЕНИЕ [6; $\$ 4]$. Пусть $\lambda \in \mathbb{R}$ - произвольное действительное число. Весовым пространством $H_{\rho}^{\lambda}=H_{\rho}^{\lambda}\left(\left\{P_{n}(x)\right\}\right)$ на $[-1,1]$ называется множество таких функций (или обобщенных функций для $\lambda<0$ ) $u(x)$, что функция

$$
u_{\lambda}(x)=\sum \underline{n}^{\lambda} \widehat{u}(n) P_{n}(x)
$$

принадлежит пространству $L_{2, \rho}$, где $\underline{n}=\max \{1, n\}$, а числа $\widehat{u}(n)$ определяются по формуле $(1.17)$ с заменой $f(x)$ на $u(x)$. Нормой функции $u(x)$ в пространстве $H_{\rho}^{\lambda}$ назовем число $\|u\|_{\lambda}^{2}=\sum_{n=0}^{\infty} \underline{n}^{2 \lambda}|\widehat{u}(n)|^{2}$. Скалярныц произведением функций $u(x)$ и $v(x)$ из пространства $H_{\rho}^{\lambda}$ назовем число $(u, v)=\sum_{n=0}^{\infty} \underline{n}^{2 \lambda} \widehat{u}(n) \widehat{v}(n)$. Относительно этого скалярного произведения пространство $H_{\rho}^{\lambda}$ становится гильбертовым.

Рассмотрим пространства $L_{2, \rho_{1}}$ и $L_{2, \rho_{2}}$ на $[-1,1]$ с

$$
\rho_{1}=\frac{1}{\sqrt{1-x^{2}}} \text { и } \rho_{2}=\sqrt{1-x^{2}} .
$$

В пространстве $L_{2, \rho_{1}}$ ортогональной системой многочленов являются многочлены

$$
\begin{array}{cc}
T_{n}^{*}(x)=a_{n} T_{n}(x), & T_{n}(x)=\cos (n \arccos x), \\
a_{n}=\frac{1}{\sqrt{\pi}}, \quad n=0, & a_{n}=\sqrt{\frac{2}{n}}, \quad n=1,2, \ldots,
\end{array}
$$

а в $L_{2, \rho_{2}}-$ многочлены

$$
\begin{aligned}
U_{n}^{*}(x)=b_{n} U_{n}(x), \quad U_{n}(x) & =\frac{\sin ((n+1) \arccos x)}{\sin (\arccos x)}, \\
b_{n}=a_{n+1}, \quad n & =0,1, \ldots .
\end{aligned}
$$

По этим пространствам построим пространства $H_{\rho_{1}}^{\lambda}$ и $H_{\rho_{2}}^{\lambda}$. Так как справедливы спектральные соотношения

$$
\frac{1}{\pi} \int_{-1}^{1} \frac{1}{\sqrt{1-x^{2}}} \frac{T_{n}^{*}(x)}{x_{0}-x} d x=-U_{n-1}^{*}\left(x_{0}\right), \quad x_{0} \in(-1,1), \quad n=1,2, \ldots,
$$

то уравнение (1.1) можно рассматривать как оператор $S_{1}$ из пространства $L_{2, \rho_{1}}$ в пространство $L_{2, \rho_{2}}$, определяемый по формуле

$$
\left(S_{1} u(x)\right)\left(x_{0}\right)=\frac{1}{\pi} \int_{-1}^{1} \frac{1}{\sqrt{1-x^{2}}} \frac{u(x) d x}{x_{0}-x} .
$$

Оператор $S_{1}$ ограничен в этой паре пространств, т.е. $S_{1} \in L\left(L_{2, \rho_{1}} ; L_{2, \rho_{2}}\right)$, и имеет индекс $\varkappa=1$. Следовательно, для любого числа $\lambda$ имеем, что оператор $S_{1}$ допускает расширение до ограниченного оператора в паре пространств $\left(H_{\rho_{1}}^{\lambda} ; H_{\rho_{2}}^{\lambda}\right)$, т.е. $S_{1} \in L\left(H_{\rho_{1}}^{\lambda} ; H_{\rho_{2}}^{\lambda}\right)$ на $[-1,1]$. 
Рассмотрим теперь функцию $\delta\left(x_{0}-q\right)$ и представим ее рядом Фурье по базису $\left\{U_{n}^{*}\left(x_{0}\right), n=0,1, \ldots\right\}$ пространства $L_{2, \rho_{2}}$, т.е. напишем формально (используя равенства (1.3) и (1.4))

$$
\begin{gathered}
\delta\left(x_{0}-q\right) \doteq \sum_{n=0}^{\infty} \widehat{\delta}_{q}(n) U_{n}^{*}\left(x_{0}\right), \\
\widehat{\delta}_{q}(n)=\int_{-1}^{1} \sqrt{1-x^{2}} \delta(x-q) U_{n}^{*}(x) d x=\sqrt{1-q^{2}} U_{n}^{*}(q), \quad n=0,1, \ldots
\end{gathered}
$$

Так как в силу определения многочлена $U_{n}\left(x_{0}\right)$ имеем $\left|\sqrt{1-q^{2}} U_{n}^{*}(q)\right| \leqslant \sqrt{2 / \pi}$, то получаем, что $\delta\left(x_{0}-q\right) \in H_{\rho_{2}}^{\lambda}$ для любого $\lambda<-1 / 2$.

Теперь аналогично функцию $\psi(x)=-\frac{1}{\pi} \frac{Q \sqrt{1-q^{2}}}{x-q}$ представим рядом Фурье по базису $\left\{T_{n}^{*}(x), n=0,1, \ldots\right\}$ пространства $L_{2, \rho_{1}}$ :

$$
\begin{aligned}
\psi(x) & =-\frac{1}{\pi} \frac{Q \sqrt{1-q^{2}}}{x-q} \doteq \sum_{n=0}^{\infty} \widehat{\psi}(n) T_{n}^{*}(x), \\
\widehat{\psi}(n) & =-\frac{Q \sqrt{1-q^{2}}}{x-q} \int_{-1}^{1} \frac{1}{\sqrt{1-x^{2}}} \frac{T_{n}^{*}(x)}{x-q} d x \\
& =-Q \sqrt{1-q^{2}} U_{n-1}^{*}(q), \quad n=1,2, \ldots, \\
\widehat{\psi}(0) & =0
\end{aligned}
$$

Таким образом, опять получаем, что функция $\psi(x) \in H_{\rho_{1}}^{\lambda}$ для любого $\lambda<-1 / 2$. Теперь, рассматривая уравнение (1.1) как оператор $S_{1}$ из пространства $H_{\rho_{1}}^{\lambda}$ в пространство $H_{\rho_{2}}^{\lambda}, \lambda<-1 / 2$, для функции $\gamma(x)$ из $(1.9)$ получаем

$$
\begin{aligned}
\frac{1}{\pi} \int_{-1}^{1} \frac{\gamma(x)}{x_{0}-x} d x & =\frac{1}{\pi} \int_{-1}^{1} \frac{1}{\sqrt{1-x^{2}}} \frac{\sum_{n=0}^{\infty} \widehat{\psi}(n) T_{n}^{*}(x)}{x_{0}-x} d x \\
& =\sum_{n=0}^{\infty} \widehat{\psi}(n) \cdot \frac{1}{\pi} \int_{-1}^{1} \frac{T_{n}^{*}(x)}{\sqrt{1-x^{2}}\left(x_{0}-x\right)} d x \\
& =Q \sum_{n=1}^{\infty} \sqrt{1-q^{2}} U_{n-1}^{*}(q) U_{n-1}^{*}\left(x_{0}\right)=Q \delta\left(x_{0}-q\right)
\end{aligned}
$$

Итак, доказана

Лемма 1.2. Для любого $\lambda<-1 / 2$, рассматривая оператор $S_{1}$ в паре пространств $\left(H_{\rho_{1}}^{\lambda} ; H_{\rho_{2}}^{\lambda}\right)$, получаем, что уравнение

$$
\begin{gathered}
\frac{1}{\pi} \int_{-1}^{1} \frac{\gamma(x) d x}{x_{0}-x}=f_{\delta, q}\left(x_{0}\right)=Q \cdot \delta\left(x_{0}-q\right), \quad q, x_{0} \in(-1,1), \\
\int_{-1}^{1} \gamma(x) d x=0
\end{gathered}
$$

имеет единственное решение $\gamma(x)$, даваемое формулой (1.9). 
ЗАмЕчАниЕ 1.1. Если в правой части уравнения (1.1) взять сумму функций $f\left(x_{0}\right)+f_{\delta, q}\left(x_{0}\right)$, где $f\left(x_{0}\right) \in L_{2, \rho_{2}}$, то все решения $\gamma(x)$ индекса 1 в паре пространств $\left(H_{\rho_{1}}^{\lambda} ; H_{\rho_{2}}^{\lambda}\right), \lambda<-1 / 2$, даются формулой (cp. [4; $\left.\left.(2.3 .30)\right]\right)$

$$
\begin{gathered}
\gamma(x)=-\frac{1}{\pi \cdot \sqrt{1-x^{2}}} \int_{-1}^{1} \frac{\sqrt{1-x_{0}^{2}} f\left(x_{0}\right) d x_{0}}{x-x_{0}}+\frac{C}{\pi \sqrt{1-x^{2}}}+\frac{1}{\pi} \frac{\sqrt{1-q^{2}}}{\sqrt{1-x^{2}}} \cdot \frac{Q}{q-x}, \\
x \in(-1,1),
\end{gathered}
$$

где функция $\gamma(x)$ удовлетворяет равенству

$$
\int_{-1}^{1} \gamma(x) d x=C
$$

Рассмотрим теперь уравнение (1.1) для решений индекса $\varkappa=-1$, т.е. обращающихся на обоих концах отрезка $[-1,1]$ в нуль. Будем рассматривать это уравнение вначале как оператор $S_{-1}$ в паре пространств $\left(L_{2, \rho_{2}} ; L_{2, \rho_{1}}\right)$. В силу спектральных соотношений

$$
\frac{1}{\pi} \int_{-1}^{1} \sqrt{1-x^{2}} \frac{U_{n}^{*}(x) d x}{x_{0}-x}=T_{n+1}^{*}\left(x_{0}\right), \quad x_{0} \in(-1,1), \quad n=0,1, \ldots,
$$

получаем, что $S_{-1} \in L\left(L_{2, \rho_{2}} ; L_{2, \rho_{1}}\right)$, и поэтому его можно расширить до ограниченного оператора $S_{-1} \in L\left(H_{\rho_{2}}^{\lambda} ; H_{\rho_{1}}^{\lambda}\right)$ для всех $\lambda \in \mathbb{R}$. Из соотношений (1.26) получаем, что для любой функции $v(x) \in H_{\rho_{2}}^{\lambda}, \lambda \in \mathbb{R}$, справедливо равенство

$$
\int_{-1}^{1} \frac{d x_{0}}{\sqrt{1-x_{0}^{2}}}\left(\frac{1}{\pi} \int_{-1}^{1} \sqrt{1-x^{2}} \frac{v(x)}{x_{0}-x} d x\right)=0
$$

Таким образом, если рассмотреть уравнение

$$
\frac{1}{\pi} \int_{-1}^{1} \frac{\gamma(x) d x}{x_{0}-x}=f\left(x_{0}\right)+f_{\delta, q}\left(x_{0}\right), \quad x_{0} \in(-1,1),
$$

где $f\left(x_{0}\right) \in L_{2, \rho_{1}}$, а $f_{\delta, q}\left(x_{0}\right)$ имеет вид $(1.2)$ и рассматривается как элемент пространства $H_{\rho_{1}}^{\lambda}, \lambda<-1 / 2$, то для разрешимости этого уравнения как операторного уравнения из $H_{\rho_{2}}^{\lambda}$ в $H_{\rho_{1}}^{\lambda}, \lambda<-1 / 2$, должно выполняться равенство

$$
\int_{-1}^{1} \frac{f(x)+f_{\delta, q}(x)}{\sqrt{1-x^{2}}} d x=0
$$

T.e.

$$
\int_{-1}^{1} \frac{f(x) d x}{\sqrt{1-x^{2}}}+\frac{Q}{\sqrt{1-q^{2}}}=0
$$

или

$$
Q=-\sqrt{1-q^{2}} \int_{-1}^{1} \frac{f(x)}{\sqrt{1-x^{2}}} d x
$$

Для численного решения уравнения (1.28) на основе идеи метода дискретных вихрей воспользуемся множествами $E=\left\{x_{k}, k=1, \ldots, n\right\}$ и $E_{0}=\left\{x_{0 j}, j=\right.$ $0,1, \ldots, n\}$, построенньми выше. Справедлива следуюшая 
ЛЕмма 1.3. Пусть функиия $f(x)$ принадлежит классу $H(\alpha)$ на $[-1,1]$ (см. $[7 ; \S 1.1])$, а функиия $f_{\delta, q}(x)$ имеет вид (1.2). Тогда решение системы линейных алгебраических уравнений

$$
\frac{1}{\pi} \gamma_{0 n}+\frac{1}{\pi} \sum_{k=1}^{n} \frac{\gamma_{n}\left(x_{k}\right) h}{x_{0 j}-x_{k}}=f\left(x_{0 j}\right)+Q \delta_{h}\left(x_{0 j}-q\right), \quad j=0,1, \ldots, n,
$$

где $\gamma_{0 n}$ - регуляризирующая переменная [7; §5.1], и значения функиии

$$
\gamma(x)=-\frac{\sqrt{1-x^{2}}}{\pi} \int_{-1}^{1} \frac{f\left(x_{0}\right) d x_{0}}{\sqrt{1-x_{0}^{2}}\left(x-x_{0}\right)}-\frac{Q}{\pi} \frac{\sqrt{1-x^{2}}}{\sqrt{1-q^{2}}} \frac{1}{x-q}, \quad x \in(-1,1)
$$

связань соотношением (1.10), в котором величина $\theta_{n}\left(x_{k}\right)$ удовлетворяет неравенствам (1.11) и (1.12).

ДокАзАТЕЛЬство. Решая систему (1.31) (см. [7; §5.1]), получаем

$$
\begin{gathered}
\gamma_{n}\left(x_{k}\right)=-\frac{1}{h} I_{-1, k}^{(n)} \sum_{j=0}^{n} \frac{1}{h} I_{-1,0 j}^{(n)} \frac{\pi\left(f\left(x_{0 j}\right)+Q \delta_{h}\left(x_{0 j}-q\right)\right) h}{x_{k}-x_{0 j}} \\
=-\frac{1}{h} I_{-1, k}^{(n)} \sum_{j=0}^{n} \frac{1}{h} I_{-1,0 j}^{(n)} \frac{\pi f\left(x_{0 j}\right) h}{x_{k}-x_{0 j}}-\frac{1}{h} I_{-1, k}^{(n)} \frac{1}{h} I_{-1,0 j_{q}}^{(n)} \frac{\pi Q}{x_{k}-q} \\
k=1, \ldots, n .
\end{gathered}
$$

Оказывается, что справедливы представления [7; § 5.1]

$$
\begin{aligned}
& \frac{1}{h} I_{-1, k}^{(n)}=\frac{1}{\pi} \sqrt{1-x_{k}^{2}}+O\left(\frac{h}{\left(1-x_{k}^{2}\right)^{1 / 2}}\right), \quad k=1, \ldots, n, \\
& \frac{1}{h} I_{-1,0 j}^{(n)}=\frac{1}{\pi} \frac{1}{\sqrt{1-x_{0 j}^{2}}}+O\left(\frac{h}{\left(1-x_{0 j}^{2}\right)^{3 / 2}}\right), \quad j=0,1, \ldots, n,
\end{aligned}
$$

из которых следует справедливость леммы 1.3. Для регуляризируюшей переменной $\gamma_{0 n}$ имеем

$$
\gamma_{0 n}=\sum_{j=0}^{n} \frac{1}{h} I_{-1,0 j}^{(n)}\left(f\left(x_{0 j}\right)+Q \delta_{h}\left(x_{0 j}-q\right)\right) h=\sum_{j=0}^{n} \frac{f\left(x_{0 j}\right) h}{\sqrt{1-x_{0 j}^{2}}}+\frac{Q}{\sqrt{1-q^{2}}}+O\left(h^{1 / 2}\right),
$$

откуда следует, что $\lim _{n \rightarrow \infty} \gamma_{0 n}=0$ тогда и только тогда, когда вьполняется условие (1.29) разрешимости уравнения (1.28) в рассматриваемом классе функций.

Покажем теперь, что функция

$$
\gamma_{q}(x)=-\frac{Q}{\pi} \frac{\sqrt{1-x^{2}}}{\sqrt{1-q^{2}}} \frac{1}{x-q}, \quad x \in(-1,1)
$$


является решением уравнения (1.28) при $f\left(x_{0}\right)=-Q / \sqrt{1-q^{2}}$ при рассмотрении этого уравнения как оператора из $H_{\rho_{2}}^{\lambda}$ в $H_{\rho_{1}}^{\lambda}, \lambda<-1 / 2$. Итак, рассмотрим функцию $\delta\left(x_{0}-q\right)$ в пространстве $H_{\rho_{1}}^{\lambda}$, используя ее разложение в ряд по многочленам $T_{n}^{*}(x)$. Имеем

$$
\begin{aligned}
\delta\left(x_{0}-q\right) & \doteq \sum_{n=0}^{\infty} \widehat{\delta}_{q}(n) T_{n}^{*}\left(x_{0}\right), \\
\widehat{\delta}_{q}(n) & =\int_{-1}^{1} \frac{1}{\sqrt{1-x^{2}}} \delta(x-q) T_{n}^{*}(x) d x=\frac{T_{n}^{*}(q)}{\sqrt{1-q^{2}}}, \quad n=0,1, \ldots
\end{aligned}
$$

Видим, что $\delta\left(x_{0}-q\right) \in H_{\rho_{1}}^{\lambda}$ для любого $\lambda<-1 / 2$. Теперь в силу спектрального соотношения (1.26) получаем, что функцию

$$
\psi(x)=-\frac{Q}{\pi \sqrt{1-q^{2}}} \cdot \frac{1}{x-q}
$$

можно представить рядом

$$
\begin{aligned}
& \psi(x)=-\frac{Q}{\pi \sqrt{1-q^{2}}} \cdot \frac{1}{x-q} \doteq \sum_{n=0}^{\infty} \widehat{\psi}(n) U_{n}^{*}(x) \\
& \widehat{\psi}(n)=-\frac{Q}{\pi \sqrt{1-q^{2}}} \cdot \int_{-1}^{1} \sqrt{1-x^{2}} \frac{U_{n}^{*}(x)}{x-q} d x=\frac{Q}{\sqrt{1-q^{2}}} T_{n+1}^{*}(q), \quad n=0,1, \ldots
\end{aligned}
$$

Поэтому для функции $\gamma(x)$ из (1.36) имеем

$$
\begin{aligned}
\frac{1}{\pi} \int_{-1}^{1} \frac{\gamma(x) d x}{x_{0}-x} & =\frac{Q}{\pi \sqrt{1-q^{2}}} \int_{-1}^{1} \sqrt{1-x^{2}} \frac{\sum_{n=0}^{\infty} T_{n+1}^{*}(q) U_{n}^{*}(x) d x}{x_{0}-x} \\
& =\frac{Q}{\pi \sqrt{1-q^{2}}} \sum_{n=0}^{\infty} T_{n+1}^{\infty}(q) \int_{-1}^{1} \frac{\sqrt{1-x^{2}} U_{n}^{*}(x) d x}{x_{0}-x} \\
& =\frac{Q}{\sqrt{1-q^{2}}} \sum_{n=1}^{\infty} T_{n}^{*}(q) T_{n}^{*}\left(x_{0}\right) .
\end{aligned}
$$

Теперь из сравнения формул (1.38) и (1.39) видно, что

$$
\begin{gathered}
\frac{1}{\pi} \int_{-1}^{1}\left(-\frac{Q}{\pi} \frac{\sqrt{1-x^{2}}}{\sqrt{1-q^{2}}}\right) \cdot \frac{1}{x-q} \cdot \frac{1}{x_{0}-x} d x=-\frac{Q}{\pi \sqrt{1-q^{2}}}+Q \delta\left(x_{0}-q\right), \\
x_{0} \in(-1,1) .
\end{gathered}
$$

Таким образом, доказана 
Лемма 1.4. Для любого $\lambda<-1 / 2$, рассматривая оператор $S_{-1}$ в nаре пространств $\left(H_{\rho_{2}}^{\lambda} ; H_{\rho_{1}}^{\lambda}\right)$, получаем, что для уравнения

$$
\frac{1}{\pi} \int_{-1}^{1} \frac{\gamma(x) d x}{x_{0}-x}=-\frac{Q}{\pi \sqrt{1-q^{2}}}+Q \delta\left(x_{0}-q\right), \quad x_{0} \in(-1,1)
$$

выполняется условие разрешимости

$$
\int_{-1}^{1} \frac{1}{\sqrt{1-x^{2}}}\left(-\frac{Q}{\pi \sqrt{1-q^{2}}}+Q \delta(x-q)\right) d x=-\frac{Q}{\sqrt{1-q^{2}}}+\frac{Q}{\sqrt{1-q^{2}}}=0
$$

и существует единственное решение $\gamma(x)$, даваемое формулой (1.36).

Наконец, рассмотрим уравнение (1.1) для решений индекса $\varkappa=0$, т.е. обрашаюшихся в нуль на одном конце и в бесконечность на другом. Для определенности будем полагать, что решение обрашается в нуль в точке $x=1$. Будем рассматривать это уравнение вначале как оператор $S_{0}$ в паре пространств $\left(L_{2, \rho_{3}} ; L_{2, \rho_{4}}\right)$, где

$$
\rho_{3}=\sqrt{\frac{1-x}{1+x}}, \quad \rho_{4}=\sqrt{\frac{1+x}{1-x}} .
$$

В пространстве $L_{2, \rho_{3}}$ системой ортогональных многочленов являются многочлены

$$
p_{n}(x)=\frac{1}{\sqrt{\pi}} \frac{T_{n+1}(x)-T_{n}(x)}{1-x}, \quad n=0,1, \ldots
$$

а в пространстве $L_{2, \rho_{4}}$ - многочлены

$$
g_{n}(x)=\frac{1}{\sqrt{\pi}}\left[U_{n}(x)-U_{n-1}(x)\right], \quad n=0,1, \ldots, \quad U_{-1}(x) \equiv 0 .
$$

В силу спектральных соотношений

$$
\begin{aligned}
\frac{1}{\pi} \int_{-1}^{1} \sqrt{\frac{1-x}{1+x}} \frac{P_{n}(x) d x}{x_{0}-x} & =\frac{1}{\pi} \int_{-1}^{1} \sqrt{\frac{1-x}{1+x}} \frac{1}{\sqrt{\pi}} \frac{T_{n+1}(x)-T_{n}(x)}{1-x} \frac{d x}{x_{0}-x} \\
& =-\frac{1}{\sqrt{\pi}}\left[U_{n}\left(x_{0}\right)-U_{n-1}\left(x_{0}\right)\right]=-g_{n}\left(x_{0}\right), \quad n=0,1, \ldots,
\end{aligned}
$$

получаем, что $S_{0} \in L\left(L_{2, \rho_{3}} ; L_{2, \rho_{4}}\right)$, и поэтому его можно расширить до ограниченного оператора $S_{0} \in L\left(H_{\rho_{3}}^{\lambda} ; H_{\rho_{4}}^{\lambda}\right)$ для всех $\lambda \in \mathbb{R}$.

Используя идеи метода дискретных вихрей и множества $E=\left\{x_{k}, k=1, \ldots, n\right\}$, $E_{0}=\left\{x_{0 j}, j=0,1, \ldots, n\right\}$, построенные выше, аналогично леммам 1.1 и $1.3 \mathrm{c}$ использованием теоремы 5.1 .1 из [ $7 ; \S 5.1]$ доказывается 
ЛЕмма 1.5. Пусть в правой части уравнения (1.1) стоит функция $f_{\delta, q}\left(x_{0}\right)$, $x_{0} \in(-1,1)$. Тогда решение системы линейных алгебраических уравнений

$$
\frac{1}{\pi} \sum_{k=1}^{n} \frac{\gamma_{n}\left(x_{k}\right) h}{x_{0 j}-x_{k}}=Q \delta_{h}\left(x_{0 j}-q\right), \quad j=1, \ldots, n
$$

и значение функиии

$$
\gamma(x)=-\frac{1}{\pi} \sqrt{\frac{1-x}{1+x}} \sqrt{\frac{1+q}{1-q}} \frac{Q}{x-q}, \quad x \in(-1,1),
$$

связаны соотношением (1.10), в котором величина $\theta_{n}\left(x_{k}\right)$ удовлетворяет неравенствам (1.11) и (1.12).

Теперь с использованием представления функции $\delta\left(x_{0}-q\right)$ рядом Фурье по системе многочленов $\left\{p_{n}(x), n=0,1, \ldots\right\}$, а функции

$$
\gamma(x)=-\frac{1}{\pi} \sqrt{\frac{1+q}{1-q}} \frac{Q}{x-q}
$$

- рядом Фурье по системе многочленов $\left\{g_{n}(x), n=0,1, \ldots\right\}$ аналогично леммам 1.2 и 1.4 доказывается

Лемма 1.6. Для любого $\lambda<-1 / 2$, рассматривая оператор $S_{0}$ в паре пространств $\left(H_{\rho_{3}}^{\lambda} ; H_{\rho_{4}}^{\lambda}\right)$, получаем, что уравнение

$$
\frac{1}{\pi} \int_{-1}^{1} \frac{\gamma(x) d x}{x_{0}-x}=Q \delta\left(x_{0}-q\right), \quad x_{0} \in(-1,1)
$$

имеет единственное решение $\gamma(x)$, даваемое формулой (1.45).

\section{§2. Сингулярное интегральное уравнение первого рода на отрезке}

Рассмотрим теперь сингулярное интегральное уравнение

$$
\frac{1}{\pi} \int_{-1}^{1} \frac{\gamma(x) d x}{x_{0}-x}+\int_{-1}^{1} K\left(x_{0}, x\right) \gamma(x) d x=f\left(x_{0}\right)+f_{\delta, q}\left(x_{0}\right), \quad x_{0} \in(-1,1)
$$

где функции $f(x)$ и $K\left(x_{0}, x\right)$ принадлежат классу $H(\alpha)$ на соответствующих множествах.

Так как характеристическое сингулярное интегральное уравнение, рассматриваемое как оператор в соответствующей паре пространств $L_{2, \rho}$, обратимо и операторы $S_{1}^{-1}, S_{-1}^{-1}, S_{0}^{-1}$, обратные к операторам $S_{1}, S_{-1}, S_{0}$, задаются соответствующими интегралами, то расширенные на соответствующую пару пространств $H_{\rho}^{\lambda}$, $\lambda \in \mathbb{R}$, обратные операторы будут задаваться теми же интегралами.

Рассмотрим теперь оператор (2.1) как оператор из $H_{\rho_{1}}^{\lambda}$ в $H_{\rho_{2}}^{\lambda}$ для решений индекса $\varkappa=1$. Будем предполагать, что если в правой части уравнения (2.1) стоит 
функция из пространства $L_{2, \rho_{2}}$, то этот оператор при условии (1.25) имеет единственное решение из $L_{2, \rho_{1}}$. Возьмем обратный к характеристическому оператору $(2.1)$ в классе функций индекса $\varkappa=1$, т.е. как оператор из $H_{\rho_{1}}^{\lambda}$ в $H_{\rho_{2}}^{\lambda}, \lambda<-1 / 2$, тогда

$$
\begin{gathered}
\gamma(x)-\frac{1}{\pi \sqrt{1-x^{2}}} \int_{-1}^{1} \frac{\sqrt{1-x_{0}^{2}} d x_{0}}{x-x_{0}} \int_{-1}^{1} K\left(x_{0}, \tau\right) \gamma(\tau) d \tau \\
=-\frac{1}{\pi \sqrt{1-x^{2}}} \int_{-1}^{1} \frac{\sqrt{1-x_{0}^{2}} f\left(x_{0}\right) d x_{0}}{x-x_{0}}+\frac{C}{\pi \sqrt{1-x^{2}}}-\frac{1}{\pi} \frac{\sqrt{1-q^{2}}}{\sqrt{1-x^{2}}} \frac{Q}{x-q} \\
x \in(-1,1), \quad x \neq q .
\end{gathered}
$$

В силу линейности уравнения (2.2) будем искать решение $\gamma(x)$ в виде

$$
\gamma(x)=-\frac{1}{\pi} \frac{\sqrt{1-q^{2}}}{\sqrt{1-x^{2}}} \frac{Q}{x-q}+\frac{\psi(x)}{\pi \sqrt{1-x^{2}}}, \quad x \in(-1,1), \quad x \neq q
$$

Тогда для функции $\psi(x)$ получаем интегральное уравнение Фредгольма второго рода

$$
\begin{gathered}
\psi(x)-\int_{-1}^{1} \widetilde{K}(x, \tau) \psi(\tau) d \tau=\lambda(x, c)+\widetilde{f}_{q}(x), \quad x \in(-1,1), \\
\lambda(x, c)=-\int_{-1}^{1} \frac{\sqrt{1-x_{0}^{2}} f\left(x_{0}\right) d x_{0}}{x-x_{0}}+C \\
\widetilde{f}_{q}(x)=-\frac{\sqrt{1-q^{2}} Q}{\pi} \int_{-1}^{1} \frac{\sqrt{1-x_{0}^{2}}}{x-x_{0}} d x_{0} \int_{-1}^{1} K\left(x_{0}, \tau\right) \frac{d \tau}{\sqrt{1-\tau^{2}}(\tau-q)}, \\
\widetilde{K}(x, \tau)=\frac{1}{\pi} \int_{-1}^{1} \frac{\sqrt{1-x_{0}^{2}}}{\sqrt{1-\tau^{2}}} \frac{K\left(x_{0}, \tau\right)}{x-x_{0}} d x_{0},
\end{gathered}
$$

которое в силу сделанных предположений имеет единственное решение. Таким образом, уравнение (2.1) при сделанњтх предположениях имеет единственное решение вида (2.3).

Аналогичные рассуждения можно провести и для решений индексов $\varkappa=-1$, $\varkappa=0$. Только теперь при $\varkappa=-1$ условие разрешимости несколько усложняется для поиска числа $Q$.

Для численного решения уравнения (2.1) можно опять применить идеи метода дискретных вихрей и рассмотреть для нахождения решений индексов $\varkappa=1,-1,0$ системы линейных алгебраических уравнений вида (1.8), (1.31) и (1.44) соответственно. Справедлива следуюшая

TЕОРема 2.1. Пусть в уравнении (2.1) функиия $f\left(x_{0}\right)$ и ядро $K\left(x_{0}, x\right)$ принадлежсат классу $H(\alpha)$ на $[-1,1] u[-1,1] \times[-1,1]$ соответственно, а функиия $f_{\delta, q}\left(x_{0}\right)$ имеет вид (1.2). Тогда решения систем линейных алгебрачческих 
уравнений

$$
\begin{aligned}
& \frac{1}{\pi} \sum_{k=1}^{n} \frac{\gamma_{n}\left(x_{k}\right) \cdot h}{x_{0 j}-x_{k}}+\sum_{k=1}^{n} K\left(x_{0 j}, x_{k}\right) \cdot \gamma_{n}\left(x_{k}\right) \cdot h \\
& \quad=f\left(x_{0 j}\right)+f_{\delta, q}\left(x_{0 j}\right), \quad j=1, \ldots, n, \\
& \frac{1}{\pi} \sum_{k=1}^{n} \frac{\gamma_{n}\left(x_{k}\right) \cdot h}{x_{0 j}-x_{k}}+\sum_{k=1}^{n} K\left(x_{0 j}, x_{k}\right) \cdot \gamma_{n}\left(x_{k}\right) \cdot h \\
& =f\left(x_{0 j}\right)+f_{\delta, q}\left(x_{0 j}\right), \quad j=1, \ldots, n-1, \\
& \sum_{k=1}^{n} \gamma_{n}\left(x_{k}\right) \cdot h=C, \quad n \\
& \frac{1}{\pi} \gamma_{0 n}+\frac{1}{\pi} \sum_{k=1}^{n} \frac{\gamma_{n}\left(x_{k}\right) \cdot h}{x_{0 j}-x_{k}}+\sum_{k=1}^{n} K\left(x_{0 j}, x_{k}\right) \cdot \gamma_{n}\left(x_{k}\right) \cdot h \\
& =f\left(x_{0 j}\right)+f_{\delta, q}\left(x_{0 j}\right), \quad j=0,1, \ldots, n,
\end{aligned}
$$

и соответствующие решения индекса $\varkappa=0,1,-1$ уравнения (2.1) связань соотношением (1.10), в котором величина $\theta_{n}\left(x_{k}\right)$ удовлетворяет неравенствам (1.11) и (1.12).

\section{§ 3. Характеристическое гиперсингулярное интегральное уравнение на отрезке}

Рассмотрим уравнение

$$
\frac{1}{\pi} \int_{-1}^{1} \frac{g(x) d x}{\left(x_{0}-x\right)^{2}}=f\left(x_{0}\right), \quad x_{0} \in(-1,1),
$$

где интеграл понимается в смысле конечной части по Адамару $[9 ; \S 1]$ :

$$
\int_{-1}^{1} \frac{g(x) d x}{\left(x_{0}-x\right)^{2}}=\lim _{\varepsilon \rightarrow 0}\left[\int_{I \backslash O\left(x_{0}, \varepsilon\right)} \frac{g(x) d x}{\left(x_{0}-x\right)^{2}}-\frac{2 g\left(x_{0}\right)}{\varepsilon}\right] .
$$

Для интеграла (3.2) справедлива формула интегрирования по частям, и поэтому уравнение $(3.1)$ в классе функций $g(x) \in H_{(0), 1}^{*}$, т.е. $g^{\prime}(x) \in H^{*}[8 ; \S 8]$ и $g(-1)=$ $g(1)=0$, эквивалентно уравнению

$$
\frac{1}{\pi} \int_{-1}^{1} \frac{g^{\prime}(x) d x}{x_{0}-x}=-f\left(x_{0}\right), \quad x_{0} \in(-1,1),
$$

при условии

$$
\int_{-1}^{1} g^{\prime}(x) d x=0
$$

Пусть теперь в уравнении (3.1) справа стоит функция $f_{\delta, q}\left(x_{0}\right)($ см. $(1.2))$, тогда уравнение (3.3) имеет своим решением функцию

$$
g^{\prime}(x)=\frac{1}{\pi} \frac{\sqrt{1-q^{2}}}{\sqrt{1-x^{2}}} \frac{Q}{x-q}, \quad x \in(-1,1)
$$


а следовательно, в силу равенства (3.4) уравнение (3.1) имеет своим решением функцию

$$
g(x)=\frac{1}{\pi} \ln \frac{|x-q|}{1-x q+\sqrt{1-x^{2}} \sqrt{1-q^{2}}}, \quad x \in(-1,1) .
$$

Для численного решения (3.6) уравнения (3.1) в случае, когда в правой части стоит функция $f_{\delta, q}\left(x_{0}\right)$, опять воспользуемся идеей метода дискретных вихрей с использованием множеств $E=\left\{x_{k}, k=1, \ldots, n\right\}$ и $E_{0}=\left\{x_{0 j}, j=0,1, \ldots, n\right\}$, построенных в $\S 1$. Справедлива

ТЕОРема 3.1. Пусть в правой части уравнения (3.1) стоит функиия $f_{\delta, q}\left(x_{0}\right), x_{0}, q \in(-1,1)$. Тогда решение системы линейных алгебрачческих уравнений

$$
\frac{1}{\pi} \sum_{k=1}^{n} g_{n}\left(x_{0 k}\right)\left[\frac{1}{x_{0 j}-x_{k+1}}-\frac{1}{x_{0 j}-x_{k}}\right]=Q \delta_{h}\left(x_{0 j}-q\right), \quad j=1,2, \ldots,
$$

и значения решения $g(x)$, даваемого формулой (3.6), уравнения (3.1) связань соотношением

$$
\left[g\left(x_{0 k}\right)-g_{n}\left(x_{0 k}\right)\right] \leqslant C h^{\lambda_{3}}, \quad \lambda_{3}>0,
$$

әде $C$ не зависит от $n$.

ДокАЗАтЕльство. Система (3.7) эквивалентна системе (1.8), если в ней заменить $\gamma_{n}\left(x_{k}\right)$ на $-g_{n}^{\prime}\left(x_{k}\right), g_{n}^{\prime}\left(x_{k}\right)=\left\lfloor g_{n}\left(x_{0 k}\right)-g_{n}\left(x_{0 k-1}\right)\right\rfloor \cdot(1 / h), k=1, \ldots, n+1$, $g_{n}\left(x_{00}\right)=g_{n}\left(x_{0 k+1}\right)=0$. Тогда для значений $-g_{n}^{\prime}\left(x_{k}\right)$ будет справедлива формула $(1.14), k=1, \ldots, n+1$. Производя численное интегрирование равенства $(1.14)$ по $m=1, \ldots, k$, т.е. воспользовавшись равенством $g_{n}\left(x_{0 k}\right)=\sum_{m=1}^{k} g_{n}^{\prime}\left(x_{m}\right) h$, в силу соотношения (1.12) получим справедливость теоремы 3.1.

\section{Список литературы}

1. Koening D. G., Falarski M.D. Aerodynamic characteristics of a large-scale model with a swept and augmented jet flap. NASA TM X 62029, 1971.

2. Бушуев В.И., Лифанов И. К. Численноерешение сингулярных интегральных уравнений в классе сингулярных функций и задача отсоса потока в аэродинамике // ЖВМ и МФ. 1986. № 10. C. 1572-1577.

3. Belotserkovsky S. M., Lifanov I. K. Metod of discrete vortices. CRC Press, 1993.

4. Лифанов И. К. Метод сингулярных интегральных уравнений и численньй эксперимент. М.: ТОО "Янус", 1995.

5. Gulyaev V. V., Lifanov I. K., Misko V.A. Mathematical model of the flow around airfoil with high-lift devices // Russian J. Numer. Anal. Math. Modelling. 1996. V. 11. № 2 . P. 155-166.

6. Вайникко Г. М., Лифанов И. К. Моделирование задач аэродинамики и дифракции волн и расширение интегралњыт операторов типа Коши на замкнутых и разомкнутых кривых // Дифференц. уравнения. 2000. Т. 36. № 9. С. 1184-1195.

7. Белоцерковский C. М., Лифанов И. К. Численные методы в сингулярных интегральных уравнениях. М.: Наука, 1985.

8. Мусхелишвили Н. И. Сингулярные интегральные уравнения. М.: Наука, 1968.

9. Анфиногенов А. Ю., Лифанов И.К., Лифанов П. И. О некоторых одномерных и двумерных интегральных уравнениях // Матем. сб. 2001. Т. 192. № 8. С. 3-46.

Институт математики Хельсинского

технологического университета, Финляндия;

Военньй авиационньй технический

университет им. Н.Е. Жуковского, г. Москва
Поступила в редакцию 10.01.2002 и 20.06 .2002 